\title{
Intraoperative Incident Dark Field Imaging of the Human Peritoneal Microcirculation
}

\author{
Zühre Uz ${ }^{a}$ e Arnoud W. Kastelein ${ }^{b}$ Dan M.J. Milstein ${ }^{c}$ Dan Liu $^{a}$ Fadi Rassam $^{a}$ \\ Denise P. Veelo $^{d}$ Jan-Paul W.R. Roovers ${ }^{b}$ Can Ince ${ }^{e}$ Thomas M. van Gulik ${ }^{a}$ \\ a Department of Surgery, Academic Medical Center, Amsterdam, The Netherlands; ${ }^{b}$ Department of Obstetrics \\ and Gynecology, Academic Medical Center, Amsterdam, The Netherlands; ' Department of Oral and Maxillofacial \\ Surgery, Academic Medical Center, Amsterdam, The Netherlands; ${ }^{\mathrm{d} D e p a r t m e n t}$ of Anesthesiology, Academic \\ Medical Center, Amsterdam, The Netherlands; ' Department of Translational Physiology, Academic Medical Center, \\ Amsterdam, The Netherlands
}

\section{Keywords}

In vivo microcirculation - Peritoneal microcirculation

Incident dark field imaging

\begin{abstract}
Background/Aims: This study describes the peritoneal microcirculation, compares quantitative parameters and angioarchitecture to the standard of sublingual microcirculatory assessment, and determines the practical feasibility of this method. Methods: Incident dark field imaging was performed of the peritoneum and sublingually to determine angioarchitecture, total and perfused vessel density (TVD and PVD), the proportion of perfused vessels (PPV), the microvascular flow index (MFI) and image acquisition time. Results: Peritoneal angioarchitecture was characterized by a quadrangular network of longitudinally oriented capillaries, often flanked by fat cells. Differences between peritoneal and sublingual microcirculation were observed with regard to TVD (peritoneum $12 \mathrm{~mm} / \mathrm{mm}^{2}$ [95\% Cl 10-14] vs. sublingual $23 \mathrm{~mm} / \mathrm{mm}^{2}$ [95\% Cl 21-25]; $p<0.0001$ ), PVD (peritoneum
\end{abstract}

\begin{tabular}{ll}
\hline KARGER & ( ) 2018 The Author(s) \\
& Published by S. Karger AG, Basel Oparger \\
E-Mail karger@karger.com & This article is licensed under the Creative Commons Attribution- \\
www.karger.com/jvr & NonCommercial-NoDerivatives 4.0 International License (CC BY- \\
NC-ND) (http://www.karger.com/Services/OpenAccessLicense). \\
Usage and distribution for commercial purposes as well as any dis- \\
tribution of modified material requires written permission.
\end{tabular}

$11 \mathrm{~mm} / \mathrm{mm}^{2}$ [95\% Cl 9-13] vs. sublingual $23 \mathrm{~mm} / \mathrm{mm}^{2}$ [95\% Cl 21-25]; $p<0.0001$ ), PPV (peritoneum 88\% [95\% Cl 79-97] vs. sublingual 99\% [95\% CI 99-100]; $p=0.014$ ), and MFI (peritoneum 3 [IQR 2.3-3.0] vs. sublingual 3 [IQR 3.0-3.0]; $p=$ $0.012)$. There was no difference in image acquisition time (peritoneum 2:34 $\min$ [95\% Cl 1:49-3:19] vs. sublingual 2:38 [95\% Cl 1:37-3:32]; $p=0.916)$. Conclusion: The peritoneal microcirculation was characterized by a low capillary density and a distinctive angioarchitecture. The possibility of peritoneal microcirculatory assessment offers promise for the study of peritoneal (patho-)physiology and (monitoring or detection of) associated diseases.

$$
\begin{aligned}
& \text { (c) } 2018 \text { The Author(s) } \\
& \text { Published by S. Karger AG, Basel }
\end{aligned}
$$

\section{Introduction}

The peritoneum lines the abdominopelvic cavity and covers the majority of visceral organs [1]. It is essential for the regulation of the intra-abdominal inflammatory response [2], exchange of peritoneal fluid [3], and preven-

Dr. Arnoud W. Kastelein

Department of Obstetrics and Gynecology

Academic Medical Center, University of Amsterdam

Meibergdreef 9, NL-1105 AZ Amsterdam (The Netherlands)

E-Mail a.w.kastelein@ amc.nl 
tion of fibrosis [4]. Many pathological processes can affect the function of the peritoneal membrane. For instance, surgical trauma or inflammation and subsequent adhesion formation and fibrosis can decrease diffusion capacity, cause pain, impair female fertility, and hamper surgical interventions [4-6]. If the peritoneum is affected by metastases of gastrointestinal or gynecological malignancies, this ultimately leads to peritoneal carcinomatosis, which is associated with a poor clinical outcome [7, 8]. In case of endometriosis, endometrial tissue can affect the pelvic peritoneum, occasionally requiring surgery because of pain and infertility [9]. The previous are examples of processes that are not sufficiently understood and for which optimal and effective treatment is often not possible. This stresses the necessity for improved understanding of peritoneal (patho-)physiology.

When peritoneal structure and function are compromised in response to pathological processes, this is expected to result in changes to the vasculature $[1,6,10]$. Generally, the peritoneum is expected to have a relatively low blood vessel density; this can however be altered in response to disease $[1,11,12]$. Therefore, improved understanding of peritoneal vascularization patterns can facilitate insight into associated pathology, detection of disease, and development of new treatment strategies [13].

Assessment of the peritoneal microcirculation should be considered a necessary first step. The introduction of handheld video microscopy imaging instruments has enabled direct visualization of the human microcirculation and creates an opportunity for imaging of the peritoneal microvasculature intraoperatively. So far, these devices were predominantly applied sublingually, to determine microvascular dysfunction in critically ill patients [14, 15]. They have also been used to assess oral mucosal wound healing and appraise the microcirculatory characteristics of different human organ surfaces such as the brain, skin, gut, intestines, conjunctiva, vagina, and liver [16-24]. This multitude of data emphasizes the importance of organ microcirculation and illustrates the broad applicability and potential advantages of these imaging modalities.

The current study aimed to use the CytoCam [25], a recently introduced third-generation handheld microscope based on incident dark field (IDF) imaging, for visualization and assessment of the human peritoneal microcirculation. Measurements were performed during hepatic surgery in patients in which the peritoneum was not affected by pathology. It is hypothesized that peritoneal microcirculation is characterized by a relatively low vessel density and a distinctive vascular architecture. In order to be able to put such remarks into context, peritoneal microangioarchitecture and related quantitative parameters were compared to the current standard of sublingual microcirculatory examination.

\section{Materials and Methods}

\section{Patients}

In this single-center observational study, men and women undergoing major liver surgery were recruited at the Department of Surgery of the Academic Medical Center of the University of Amsterdam. Surgical procedures were performed in accordance with established institutional protocols and standards of care. The study complied with ethical principles and appropriate regulatory requirements of the European Union and the Netherlands. All patients received full explanation of the study procedures and provided informed consent. The protocol was reviewed and approved by the Institutional Review Board of the Academic Medical Center under number W17_259.

\section{Hemodynamic Parameters}

Hemodynamic parameters such as heart rate, arterial blood pressure and peripheral capillary oxygen saturation were acquired. Stroke volume and central venous pressure parameters were assessed with the FloTrac system (FloTrac, Edwards Lifesciences, Irvine, CA, USA) [26]. Arterial blood gas samples were collected to determine hemoglobin, $\mathrm{pH}$ and oxygen hemoglobin.

\section{Microcirculatory Imaging}

Peritoneal and sublingual microcirculation assessments were performed using IDF imaging (CytoCam Video Microscope System, Braedius Medical, Huizen, the Netherlands). This technique has been described extensively elsewhere $[25,27]$. In summary, the CytoCam uses green light $(530 \mathrm{~nm})$ produced from a ring of lightemitting diodes arranged near the tip of the probe. This green light epi-illuminates the tissue of interest and is absorbed by oxygenated and deoxygenated hemoglobin in red blood cells. Unabsorbed light is scattered into the surrounding tissues as red blood cells appear as dark globules and can be recorded flowing within the lumen of the microvasculature. This results in sharp contour visualization of the microcirculation. The CytoCam is a digital computer-controlled camera that is lightweight $(120 \mathrm{~g})$ and shaped like a pen (length $220 \mathrm{~mm}$, diameter $23 \mathrm{~mm}$ ). It uses high-brightness green light-emitting diodes with a short illumination pulse time ( $2 \mathrm{~ms}$ ) and a high spatial (14 megapixels) and temporal (60 fps) resolution. The combination of a factor 4 optical magnification and a large image area provides a field of view of $1.55 \times 1.16 \mathrm{~mm}$. The optical system provides an optical resolution of more than 300 lines $/ \mathrm{mm}$. The camera is connected to a device controller based on a powerful medical grade computer. Video clips are directly saved as digital AVI-DV files to a hard drive [25].

\section{Data Acquisition}

Measurements were performed 10 min after laparotomy, before the start of the surgery. Three peritoneal measurements were performed on the right lateral peritoneal side of the abdominal cavity. The region of interest was at least $20 \mathrm{~cm}$ away from the laparotomy incision site. During peritoneal measurements, the 
Fig. 1. Peritoneal microcirculation in automated vascular analysis (AVA) analysis. The proportion of perfused vessels (PPV), the perfused vessel density (PVD), and total vessel density (TVD) are determined by AVA software, as shown in this image. The PPV is calculated by scoring all vessels with a flow score greater than 1 . PVD can be calculated by multiplying TVD and PPV. Both PVD and TVD are expressed as millimeters per square millimeter. Each score was determined for small microvessels with a cutoff diameter of $25 \mu \mathrm{m}$. The red lines show the vessel wall, the yellow lines show the vessel centerline. The numbers on the vessels show the flow within each vessel.

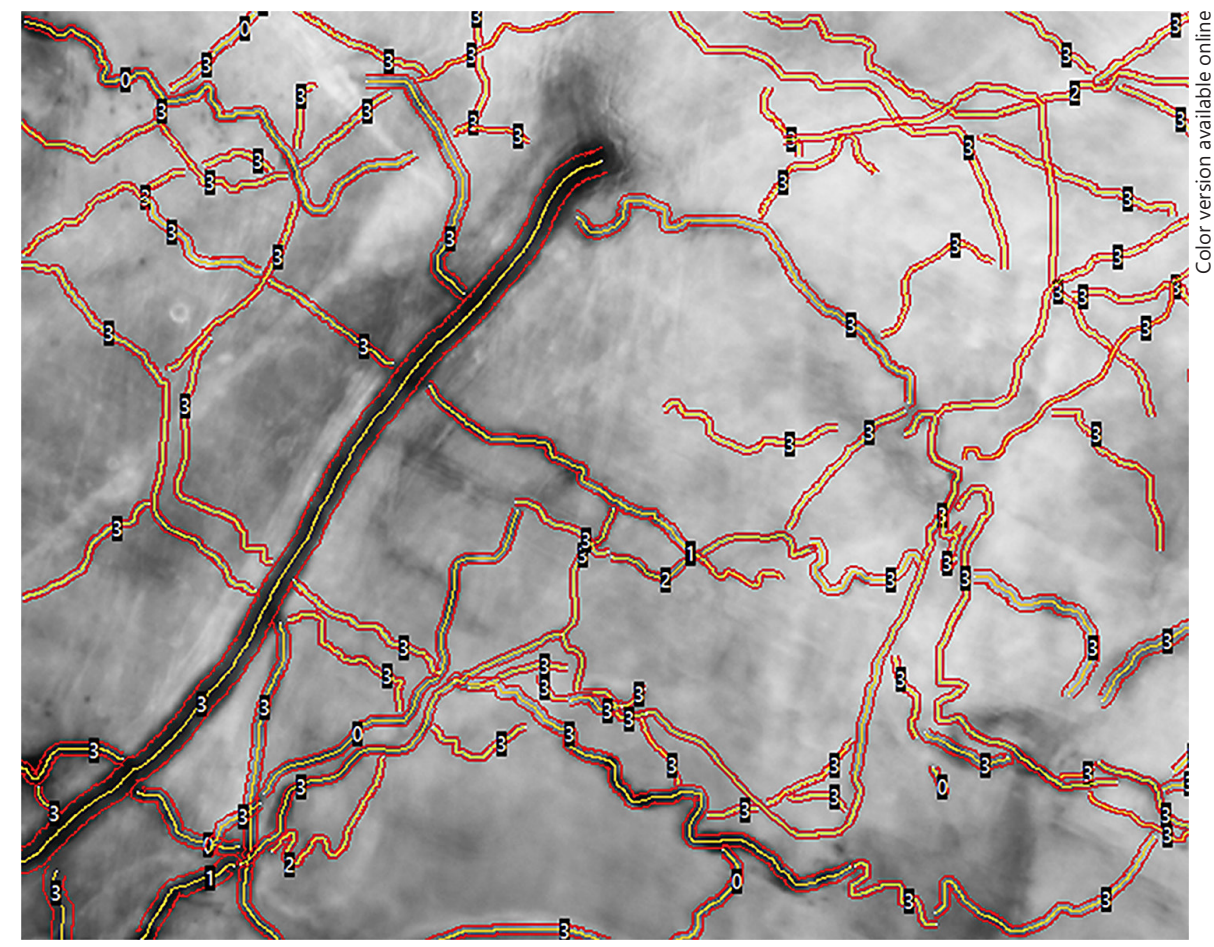

CytoCam was covered with a laparoscopy camera cover (Camera Cover, Microtek Medical BV, Zutphen, the Netherlands) to create a sterile work field. The camera was placed into contact with the peritoneal surface gently and perpendicularly, in order to prevent pressure-induced artifacts. Subsequently, 3 sublingual measurements were performed according to a standardized procedure [28]. The sublingual microcirculation was assessed in the floor of the mouth, between the lingual frenulum and the sublingual fold.

\section{Assessment of Angioarchitecture}

The layout of the peritoneal and sublingual microcirculation was described and compared. Peritoneal angioarchitecture was examined and classified as 1 of 3 types of vascular patterns, as validated and described by Weber et al. [23]. These vascular patterns include the appearance of capillary loops (score 1), the appearance of capillary loops in combination with capillary networks (score 2), and the appearance of only capillary networks (score 3 ).

\section{Quantifying Microcirculation and Comparison of}

Microcirculatory Parameters

Tissue perfusion depends on the number, distribution, and diameters of the capillaries in combination with blood viscosity and driving pressure across the capillaries. The two main hemodynamic principles governing oxygen supply to tissue are convection and diffusion. Convection is quantified by flow, and diffusion is quantified by the density of the perfused (= functional) microvessels. Flow is calculated by scoring the predominant type of flow across the microvasculature; this generates the microvascular flow index (MFI), a semiquantitative score ranging from 0 (no flow) to 3 (continuous flow) [29]. To quantify diffusion, the total and perfused vessel densities (TVD and PVD, respectively) and the proportion of perfused vessels (PPV) were determined. The PPV was calculated by scoring all vessels with a flow score greater than 1; see Figure 1. PVD can be calculated by multiplying TVD by PPV. Both TVD and PVD are expressed in millimeters per square millimeter. Each score was determined for small microvessels with a cutoff diameter of $25 \mu \mathrm{m}$.

\section{Practical Feasibility}

Image acquisition time was recorded and used as an indicator of practical feasibility. This feasibility was defined as the time it took to acquire 3 stable video sequences of the microcirculation. This applied to both peritoneal and sublingual measurements.

\section{Image Analysis}

After image acquisition, video clips were saved to a hard drive, and subsequent image analysis was performed off-line and blinded by the 2 investigators (Z.U. and A.W.K.). Aforementioned parameters TVD, PVD, PPV, and MFI were determined by softwareassisted analysis (AVA v3.2; Automated Vascular Analysis, Microvision, The Netherlands).

\section{Statistical Analysis}

Descriptive statistics was used to present the demographic variables. Non-Gaussian data sets are presented as medians and interquartile ranges (IQR), and normal Gaussian data sets are presented as means \pm standard deviation. A nonparametric test was used for MFI (Wilcoxon signed-rank) and a paired $t$ test was used for all other data. A 2 -sided $p$ value $<0.05$ was considered statistically significant. All data were analyzed using SPSS, version 23.0 for Windows (IBM Corp., Armonk, NY, USA). 
Table 1. Baseline characteristics

\begin{tabular}{lc}
\hline Sex (male/female), $n$ & $13 / 5$ \\
Median age, years (IQR) & $68(55-73)$ \\
Median body mass index (IQR) & $24.4(21.3-27.6)$ \\
Smoker, $n(\%)$ & $2(11.1)$ \\
Diabetes, $n(\%)$ & $3(16.7)$ \\
Hypertension, $n(\%)$ & $3(16.7)$ \\
COPD, $n(\%)$ & $0(0)$ \\
Pathology, $n(\%)$ & \\
CRLM & $2(11.1)$ \\
PHC & $4(22.2)$ \\
CGC & $9(50)$ \\
HCC & $1(5.6)$ \\
LA & $15.6)$ \\
NET & $1(5.6)$ \\
Procedures, $n$ (\%) & \\
Right hemihepatectomy & $9(50)$ \\
Left hemihepatectomy & $7(38.8)$ \\
Resection of liver segments 4 and 8 & $1(5.6)$ \\
Cholecystectomy & $1(5.6)$ \\
\hline
\end{tabular}

Data presented as medians or absolute numbers. COPD, chronic obstructive pulmonary disease; CRLM, colorectal liver metastasis; PHC, perihilar cholangiocarcinoma; CGC, cholangiocarcinoma; HCC, hepatocellular carcinoma; LA, liver adenoma; NET, neuroendocrine tumor.

Table 2. Hemodynamic parameters

\begin{tabular}{lc}
\hline Systemic & \\
Heart rate, beats/min & $73 \pm 12$ \\
Systolic arterial pressure, mm Hg & $106 \pm 21$ \\
Diastolic arterial pressure, $\mathrm{mm} \mathrm{Hg}$ & $58 \pm 11$ \\
Mean arterial pressure, mm Hg & $73 \pm 10$ \\
Stroke volume, mL/beat & $66 \pm 17$ \\
Central venous pressure, mm Hg & $7 \pm 4$ \\
Peripheral oxygen saturation, \% & $99 \pm 1$ \\
Core temperature, ${ }^{\circ} \mathrm{C}$ & $36.4 \pm 0.4$ \\
Blood & \\
Hemoglobin, mmol/L & $7.8 \pm 0.9$ \\
pH & $7.40 \pm 0.04$ \\
Oxygen hemoglobin, $\%$ & $97.6 \pm 0.9$ \\
\hline
\end{tabular}

Data are presented as means \pm SD. Stroke volume: measurement obtained from 14/18 patients.

\section{Results}

Patient Characteristics and Hemodynamic Parameters Eighteen patients aged 32-78 years were enrolled in this study. The majority of patients (50\%) were diagnosed with a cholangiocarcinoma for which they underwent a left or right hemihepatectomy (89\%). Baseline character- istics of patients are presented in Table 1. Table 2 summarizes the hemodynamic conditions during surgery. A total of 108 measurements were obtained; 6 measurements were performed in each patient, of which 3 were on the peritoneum and 3 sublingually. Of these measurements, 10 peritoneal (9\%) and 8 sublingual (7\%) measurements were excluded for analysis due to poor image quality; the final data set was based on a total 90 measurements.

\section{Assessment of Angioarchitecture and \\ Morphological Features: Peritoneal versus \\ Sublingual Microcirculation}

Peritoneal Microcirculation

The peritoneal microcirculation is characterized by a quadrangular network of large, parallel vessels and their longitudinally orientated capillary branches (Fig. 2). The capillaries have a tortuous structure and make longer distances without connecting to other capillaries compared to the sublingual capillaries. The peritoneal microcirculation can be classified as a score 3 angioarchitecture: a vascular network is observed without the appearance of capillary loops. The peritoneal microcirculation was often flanked by fatty tissue; fat cells were therefore seen and appeared as large globular transparent structures. The quantity of fat cells varied between patients and was not consistent overall. The peritoneal microcirculation was flanked by fat cells in 10 (53\% of the study population) patients. In 4 (21\% of the study population) patients $100 \%$ of the field of view showed fat cells, and in 6 (32\% of the study population) patients less than $50 \%$ of the field of view showed fat cells. Regardless of the presence of fat cells, microcirculatory assessment of the peritoneal microvasculature was easily possible due to their transparent appearance.

\section{Sublingual Microcirculation}

A sample of the sublingual microcirculation obtained with the CytoCam is shown in Figure 3. The large vessels of the microcirculation (diameter $>25 \mu \mathrm{m}$ ) appear curly in structure. The capillaries had a less tortuous structure and make shorter distances with more interconnections to other capillaries as compared to the peritoneal microcirculation. The sublingual microcirculation also showed capillary loops (type 2 angioarchitecture); these loops primarily appeared in the most superficial layers just beneath the subepithelium of the mucosa, the papillary layer. These capillary loops do not appear in the deeper layers of the sublingual mucosa beyond the papillary layer, the microcirculation of this 
Fig. 2. IDF image of the peritoneal microcirculation. A screenshot from a CytoCam video clip of the peritoneal microcirculation. Quadrangular network of large parallel vessels (LV) and their longitudinally orientated tortuous capillary (C) branches. The peritoneal microcirculation is often flanked by fat cells (FC).

Fig. 3. IDF image of the sublingual microcirculation. A screenshot from a CytoCam video clip of the sublingual microcirculation. The large vessels (LV) of the microcirculation (diameter $>25 \mu \mathrm{m}$ ) show a curly structure. The capillaries (C) are randomly organized with the appearance of capillary vessel loops (VL) and make short distances that interconnect with other capillary vessels.
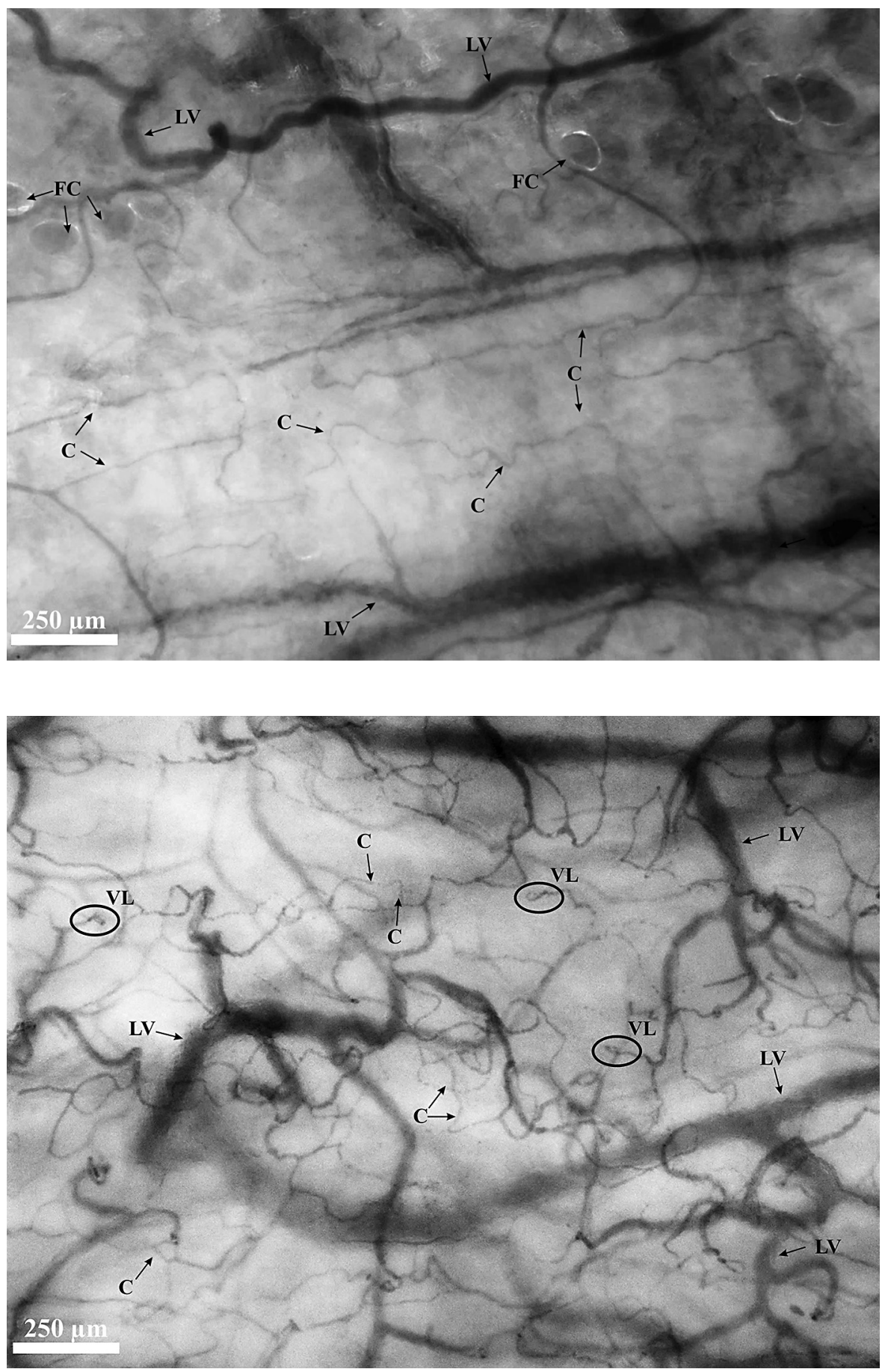

reticular layer is of a network type (type 3 angioarchitecture). According to the image quality score (17), only $30 \%$ of the image should contain capillary loops for a good quality sublingual microcirculatory assessment. These capillary loops were not observed in the peritoneal microcirculation.

\section{Microcirculatory Parameters}

The peritoneal microcirculation was quantitatively characterized by low microvascular densities (Table 3 ). It showed a significantly lower TVD (peritoneum $12 \mathrm{~mm} /$ $\mathrm{mm}^{2}$ [95\% CI 10.4-13.6] vs. sublingual $23 \mathrm{~mm} / \mathrm{mm}^{2}$ [95\% CI 21.1-24.7]; $p<0.0001$ ) and PVD (peritoneum 11 
Table 3. Microcirculatory parameters and image acquisition

\begin{tabular}{llllc}
\hline & Peritoneum & Sublingual & Difference $(95 \% \mathrm{CI})$ & $p$ value \\
\hline Median MFI (IQR) & $3(2.3-3.0)$ & $3(3.0-3.0)$ & 0 & 0.012 \\
PPV, \% & 88 & 99 & $-11(-20.4$ to -2.6$)$ & 0.014 \\
TVD, $\mathrm{mm} / \mathrm{mm}^{2}$ & 12.0 & 22.9 & $-10.9(-13.5$ to -8.4$)$ & $<0.0001$ \\
PVD, $\mathrm{mm} / \mathrm{mm}^{2}$ & 10.6 & 22.7 & $-12.1(-14.9$ to -9.1$)$ & $<0.0001$ \\
Image acquisition, min & $2: 34$ & $2: 38$ & $-0: 04(-1: 09$ to $1: 02)$ & 0.916 \\
\hline
\end{tabular}

Data presented as means, unless indicated otherwise. CI, confidence interval (difference of the means); MFI, microvascular flow index; PPV, proportion of perfused vessels; TVD, total vessel density; PVD, perfused vessel density.

$\mathrm{mm} / \mathrm{mm}^{2}$ [95\% CI 8.6-12.6] vs. sublingual $23 \mathrm{~mm} / \mathrm{mm}^{2}$ [95\% CI 20.9-24.5]; $p<0.0001)$. Moreover, differences between sublingual and peritoneal microcirculation were also observed with regard to PPV (peritoneum 88\% [95\% CI 78.5-96.6] vs. sublingual 99\% [95\% CI 98.5-99.7]; $p=$ 0.014 ) and MFI (peritoneum 3 [IQR 2.3-3.0] vs. sublingual 3 [IQR 3.0-3.0]; $p=0.012$ ). No significant differences with regard to image acquisition (i.e., time to 3 stable measurement acquisitions) were observed (peritoneum 2:34 min [95\% CI 1:49-3:19] vs. sublingual 2:38 [95\% CI 1:37-3:32]; $p=0.916$ ).

In the oldest patient (79 years old) the peritoneal microcirculation was characterized by a lower microvascular density (peritoneal microcirculation: TVD $11.9 \mathrm{~mm} /$ $\mathrm{mm}^{2}$, PVD $11 \mathrm{~mm} / \mathrm{mm}^{2}$, PPV 92.9\%, MFI 2.7; sublingual microcirculation: TVD $27 \mathrm{~mm} / \mathrm{mm}^{2}$, PVD $26.2 \mathrm{~mm} /$ $\mathrm{mm}^{2}$, PPV 97\%, MFI 3) when compared to the peritoneal microvascular density of the youngest patients (33 years old) (peritoneal microcirculation: TVD $19.2 \mathrm{~mm} /$ $\mathrm{mm}^{2}$, PVD $18 \mathrm{~mm} / \mathrm{mm}^{2}$, PPV 94\%, MFI 2.7; sublingual microcirculation: TVD $25.4 \mathrm{~mm} / \mathrm{mm}^{2}$, PVD $25.4 \mathrm{~mm} /$ $\mathrm{mm}^{2}$, PPV 100\%, MFI 3).

When analyzing the microcirculatory parameters in groups with different intraoperative hemodynamic parameters (lower and higher median for stroke volume and central venous pressure), no significant differences between the groups were observed.

\section{Discussion}

This study is the first to report on human peritoneal microcirculation assessed intraoperatively with IDF imaging. When compared to the sublingual microcirculation, assessment reveals significantly lower microvascular density and flow parameters, different morphological characteristics, and equal image acquisition times for each tissue. In order to discover the clinical value of the peritoneal microcirculation and to better understand the (patho-)physiology associated with the peritoneum, the ability to measure the peritoneal microcirculation is a necessary first step. These results demonstrate that assessment of peritoneal quantitative microcirculatory parameters and microcirculatory architecture is feasible, providing an opportunity for future research.

Within the scope of this study, the peritoneal tissue revealed a significantly lower microvessel density when compared to the sublingual microcirculation. This difference in density parameters (TVD, PVD) is most likely due to a different anatomy. The submesothelial stroma contains the peritoneal vasculature, of which vessels with a diameter of $<100 \mu \mathrm{m}$ (i.e., arterioles, capillaries, and venules) form the peritoneal microcirculation [1]. The vasculature is organized in a single layer and the microvessels are arranged relatively spaciously, resulting in a low vessel density. This low vessel density possibly affects pathological processes such as the restrictive growth of metastases on the peritoneal membrane $[8,10]$. Interestingly, not only the density, but also the peritoneal flow parameters (PPV, MFI) were significantly lower when compared to the sublingual microcirculation.

In another study by de Bruin et al. [17], sidestream dark field imaging was used to examine and measure the microcirculation of the intestinal serosa. This study also revealed lower density parameters when compared to the sublingual microcirculation. However, the authors do not describe lower flow parameters. Hypothetically, the lower PPV and MFI could be the consequence of congestive pressure caused by the tip of the CytoCam. However, such pressure would be noticed by the researcher operat- 
ing the device. This would be visible as compression of larger vessels and consequently as absence of flow in these vessels. No absence of flow was observed in the larger vessels. Another explanation might be the time point of the measurements. In our study, the measurements were obtained just after the laparotomy skin incision, whereas de Bruin et al. obtained measurements at the end of the surgery. This might lead to redistributed flow in the microcirculation. However, since our results regarding quantitative parameters of the sublingual microcirculation are in agreement with previous studies [19, 20, 25, 28], it seems that these differences cannot be attributed to the time point at which the measurements were performed.

The time for image acquisition was similar for peritoneal and sublingual assessments. The region of interest was easily accessible at both surfaces and there were no major movements that interfered with the stability of the device. In the case of sublingual assessments, removal of excessive saliva to avoid content artifacts took longest and determined the time to acquire a sublingual image. The difficulty that determined the time to acquire a peritoneal image was pressure artifacts. Use of an image or surface contact stabilizer, described previously by Balestra et al. [30] and validated by de Bruin et al. [31], could help to avoid such pressure artifacts. This contact stabilizer is attached to the tip of the device as a ring and adheres to the region of interest by applying a mild negative pressure. The surface of the imaging probe tip does not adhere to the surface and protects it from pressure artifacts and movements.

Some points of consideration should be addressed regarding study design and practicality. Firstly, the assessment of the peritoneal microcirculation with IDF imaging is only possible when patients undergo laparotomy. Therefore, these measurements can only be performed in a limited selection of patients. This illustrates a practical disadvantage of IDF imaging. With the devices that are currently available, intra-abdominal measurements can only be performed after laparotomy. Possibly, development of a laparoscopic device can facilitate future research and the clinical application of measuring intraabdominal microcirculation during less invasive (laparoscopic) surgical procedures. On the other hand, it should be taken into account that the increased intra-abdominal pressure during laparoscopic surgery, i.e. the prolonged mechanical forces pushing against the peritoneum, could affect the peritoneal microcirculation.

Secondly, in an ideal situation, measurements would have been performed in a healthy control group. Nevertheless, we considered patients with liver pathology a rel- atively suitable patient population for measurements of unaffected peritoneum. In contrast to the visceral peritoneal veins that drain into the portal vein, parietal peritoneal veins drain directly into the vena cava, and flow is therefore not expected to be affected by liver pathology [32].

Thirdly, the measurements were performed during liver surgery in patients under general anesthesia. Some literature suggests this could cause anesthesia-induced tissue perfusion changes and affect microcirculatory parameters [33]. Therefore, the measurements of the peritoneal and sublingual microcirculation were performed at the very beginning of the surgery, in order to minimize the effects of the surgery and the general anesthesia.

As mentioned above, this is the first study to report on the human peritoneal microcirculation assessed with IDF imaging. It provides unique data on in vivo dynamic and quantitative microvascular parameters and microvascular architecture. Such assessment in a pathological context should be considered a next step in future research regarding peritoneal tissue; it could facilitate the detection of pathology (peritoneal metastasis), assess reaction to treatment (the development of fibrosis after peritoneal dialysis, the effect of hyperthermal intraperitoneal chemotherapy) or contribute to improved understanding of the pathophysiology of peritoneum-associated disease (peritoneal carcinomatosis, endometriosis).

In conclusion, IDF imaging is a promising technique for the visualization and assessment of the peritoneal microcirculation. Imaging of the peritoneal membrane reveals a distinctive microvascular layout in which angioarchitecture is characterized by a quadrangular network of longitudinally oriented capillaries, often flanked by fat cells. Microvascular density and flow parameters are significantly lower than parameters measured in the sublingual microcirculation.

\section{Acknowledgments}

The authors would like to express their gratitude to all subjects willing to participate in this research. They would also like to thank operating room personnel for their participation and facilitation of researchers performing measurements.

\section{Disclosure Statement}

Prof. Ince has developed SDF imaging and is listed as inventor on related patents commercialized by MicroVision Medical (MVM) under a license from the Academic Medical Center. He receives no royalties or benefits from this license. He has been a 
consultant for MVM in the past, but has not been involved with this company for more than 5 years now and holds no shares or stock. Braedius Medical, a company owned by a relative of Prof. Ince, has developed and designed a handheld microscope called CytoCam-IDF imaging. Prof. Ince has no financial relation with
Braedius Medical of any sort, i.e., never owned shares, or received consultancy or speaker fees from Braedius Medical. Prof. Ince runs an Internet site, which offers services (e.g. training, courses, analysis) related to clinical microcirculation. The other authors declare that they have no competing interests.

\section{References}

1 Van Baal JO, et al: The histophysiology and pathophysiology of the peritoneum. Tissue Cell 2017;49:95-105.

$\checkmark 2$ Topley N, Williams JD: Role of the peritoneal membrane in the control of inflammation in the peritoneal cavity. Kidney Int Suppl 1994; 48:S71-S78.

-3 Fedorko ME, Hirsch JG: Studies on transport of macromolecules and small particles across mesothelial cells of the mouse omentum. I. Morphologic aspects. Exp Cell Res 1971;69: 113-127.

4 De Lima SM, et al: Inflammation, neoangiogenesis and fibrosis in peritoneal dialysis. Clin Chim Acta 2013;421:46-50.

5 Gomel V, Koninckx PR: Microsurgical principles and postoperative adhesions: lessons from the past. Fertil Steril 2016;106:10251031.

6 Koninckx PR, et al: Role of the peritoneal cavity in the prevention of postoperative adhesions, pain, and fatigue. Fertil Steril 2016;106: 998-1010.

7 Lee K, Tavassoli FA, Prat J: Tumors of the ovary and peritoneum; in Tavassoli FA, Devilee P (eds): World Health Organization Classification of Tumours: Pathology and Genetics of Tumours of the Breast and Female Genital Organs. Lyon, IARC Press, 2003, p 117.

-8 Lemoine L, Sugarbaker P, Van der Speeten K: Pathophysiology of colorectal peritoneal carcinomatosis: role of the peritoneum. World J Gastroenterol 2016;22:7692-7707.

9 Mahmood TA, Templeton A: Prevalence and genesis of endometriosis. Hum Reprod 1991; 6:544-549.

10 Solass W, et al: Functional vascular anatomy of the peritoneum in health and disease. Pleura Periton 2016;1:145-158.

11 Blackburn SC, Stanton MP: Anatomy and physiology of the peritoneum. Semin Pediatr Surg 2014;23:326-330.
12 Rippe B, Rosengren BI, Venturoli D: The peritoneal microcirculation in peritoneal dialysis. Microcirculation 2001;8:303-320.

13 Flessner MF, Lofthouse J, Zakaria ER: Improving contact area between the peritoneum and intraperitoneal therapeutic solutions. J Am Soc Nephrol 2001;12:807-813.

14 Spronk PE, et al: Nitroglycerin in septic shock after intravascular volume resuscitation. Lancet 2002;360:1395-1396.

15 Goedhart PT, et al: Sidestream dark field (SDF) imaging: a novel stroboscopic LED ring-based imaging modality for clinical assessment of the microcirculation. Opt Express 2007;15:15101-15114.

16 Mathura KR, Bouma G, Ince C: Abnormal microcirculation in brain tumours during surgery. Lancet 2001;358:1698-1699.

17 de Bruin AF, et al: Sidestream dark field imaging of the serosal microcirculation during gastrointestinal surgery. Colorectal Dis 2016; 18:O103-O110.

18 Klijn E, et al: Conjunctival microcirculation in patients with traumatic brain injury. Crit Care 2008;12(suppl 2):P106-P106.

19 Edul VSK, et al: Dissociation between sublingual and gut microcirculation in the response to a fluid challenge in postoperative patients with abdominal sepsis. Ann Intens Care 2014; 4:39-39.

20 Boerma EC, et al: Disparity between skin perfusion and sublingual microcirculatory alterations in severe sepsis and septic shock: a prospective observational study. Intens Care Med 2008;34:1294-1298.

21 Puhl G, et al: Noninvasive in vivo analysis of the human hepatic microcirculation using orthogonal polorization spectral imaging. Transplantation 2003;75:756-761.

22 De Backer D, et al: Microvascular blood flow is altered in patients with sepsis. Am J Respir Crit Care Med 2002;166:98-104.

$\checkmark 23$ Weber MA, et al: Vaginal microcirculation: non-invasive anatomical examination of the micro-vessel architecture, tortuosity and capillary density. Neurourol Urodyn 2015;34: 723-729.
24 Lindeboom JA, et al: Influence of the application of platelet-enriched plasma in oral mucosal wound healing. Clin Oral Implants Res 2007;18:133-139.

25 Aykut G, et al: Cytocam-IDF (incident dark field illumination) imaging for bedside monitoring of the microcirculation. Intens Care Med Exp 2015;3:40.

26 Manecke GR: Edwards FloTrac sensor and Vigileo monitor: easy, accurate, reliable cardiac output assessment using the arterial pulse wave. Expert Rev Med Devices 2005;2: 523-527.

27 Hutchings S, Watts S, Kirkman E: The Cytocam video microscope. A new method for visualising the microcirculation using incident dark field technology. Clin Hemorheol Microcirc 2016;62:261-271.

28 Ocak I, Kara A, Ince C: Monitoring microcirculation. Best Pract Res Clin Anaesthesiol 2016;30:407-418.

29 Boerma EC, et al: Quantifying bedside-derived imaging of microcirculatory abnormalities in septic patients: a prospective validation study. Crit Care 2005;9:R601-R606.

30 Balestra GM, et al: Improvement of sidestream dark field imaging with an image acquisition stabilizer. BMC Medical Imaging 2010;10:15.

31 de Bruin AF, et al: Use of an image acquisition stabilizer improves sidestream dark field imaging of the serosa during open gastrointestinal surgery. J Vasc Res 2016;53:121-127.

32 Khanna R, Krediet RT: Chapter 4: the peritoneal microcirculation in peritoneal dialysis; in Khanna R, Krediet RT (eds): Nolph and Gokal's Textbook of Peritoneal Dialysis, ed 3. New York, Springer, 2009.

33 Turek Z, et al: Anesthesia and the microcirculation. Semin Cardiothorac Vasc Anesth 2009;13:249-258. 